\title{
MPC Based Handling Qualities Assessment for a Transportation Aircraft with Failed Actuators
}

\author{
Lunlong Zhong ${ }^{1}$ and Félix Mora-Camino ${ }^{2}$ \\ ENAC, MAIAA, Univ. de Toulouse, F-31055 Toulouse, France
}

\begin{abstract}
The objective of this study is to contribute to the evaluation of the remaining handling qualities of a transportation aircraft in the situation in which some aerodynamic actuators have failed. The maneuverability issue is in this study concentrated on the control of the three rotations along the main axis of a transportation aircraft. The solution approach adopted here is to perform at first an off-line study using a realistic flight simulation model of the aircraft to design a controller for the deflection of the remaining actuators with the objective of performing in a nominal way the considered reference maneuvers. When the control objective cannot be achieved, downgraded handling qualities are identified. The offline controller design approach follows the main steps of classical Model Predictive Control (MPC). The remaining of this paper describes the way this control approach has been developed to produce a useful tool to estimate the remaining handling qualities of an aircraft with failed actuators.
\end{abstract}

$\begin{array}{lll} & & \text { Nomenclature } \\ \rho & = & \text { density of air, } \mathrm{kg} / \mathrm{m}^{3} \\ \alpha & = & \text { angle of attack, } \mathrm{rad} \\ \beta & = & \text { sideslip angle, } \mathrm{rad} \\ V & = & \text { airspeed, } \mathrm{m} / \mathrm{s} \\ \delta_{p}, \delta_{q}, \delta_{r} & = & \text { roll, pitch, yaw actuator deflections, } \mathrm{rad} \\ \mathrm{p}, q, \mathrm{r} & = & \text { roll, pitch, yaw rates, } \mathrm{rad} / \mathrm{s} \\ C_{l}, C_{m}, C_{n} & = & \text { roll, pitch, yaw dimensionless aerodynamic coefficients }\end{array}$

\section{Introduction}

$\mathrm{T}$ he objective of this study is to contribute to the evaluation of the remaining maneuverability of a transportation aircraft in the situation in which some aerodynamic actuators have failed. It is supposed here that a fault detection and identification (FDI) module will be available on-line to detect the fault and identify exactly the faulty actuator and its remaining performances, if any, so that it is considered in this study that the current state of operation of each actuator is known. The design characteristics and operations principles of such FDI module are not discussed in this paper, however main achievements in this field can be found in Refs. 1, 2. A fault free actuator will be considered to follow a nominal model with unchanged level and rate constraints. A failed actuator will be either stuck, floating or partially downgraded (longer response time, limited operation domain). The maneuverability issue is in this study concentrated on the control of the three rotations along the main axis of a transportation aircraft, since they are basic for stabilization, attitude control and further maneuvers. These rotations are mainly the result of the aerodynamic moments created by the deflection of the main and secondary aerodynamic actuators.

It is of major interest, once an actuator failure, or more, has been detected and identified to known what are the remaining handling performances. This will be particularly the case with the angular rate control system. Since it is considered that in fault free conditions, targets values for the angular rates should be reached according to nominal linear dynamics, the question is to know if this will be still possible, or if these target values will be achieved with additional delay or if these target values will remain out of range. The solution approach adopted in this study is to perform at first an off-line study using a realistic flight simulation model of the aircraft to design a controller for the

\footnotetext{
${ }^{1}$ PhD student at MAIAA, Automation Research Group, ENAC, lunlong.zhong@enac.fr

${ }^{2}$ Professor, ENAC, felix.mora@enac.fr
} 
deflection of the remaining actuators with the objective of performing in a nominal way reference maneuvers. When considering a particular maneuver which can be no more performed nominally, the adoption of this control approach will turn possible to assess feasible downgraded maneuvers. The off-line controller design approach adopted at this step of our study with the above objective and which is described in this paper, follows the main steps of classical MPC control using as test bed a realistic flight simulation model of the considered aircraft. Then, by performing a systematic generation of possible actuators failure scenarios where some effectiveness over each axis remains, a full panorama of the handling qualities of the aircraft can be built and structured as a knowledge base.

Then it appears opportune to include in a fault tolerant control structure, next to an FDI module, a supervision module associated to the knowledge base and whose main function is to decide if the fault situation can be managed as a fail-operational or a fail-passive situation. In the first case, through an actuator reassignment module, see Refs. 3-5, the current flight condition (manual or auto flight modes) at the occurrence of the fault will be maintained as well as the nominal handling performances of the aircraft, since the remaining active actuators are found able to produce timely the required moments to perform nominal maneuvers. Figure 1 (a) displays the fail-operational case with a running autopilot. Otherwise, Figure 1 (b), to insure a fail-passive situation, flight control will return to manual mode through the actuator reassignment module and with the help of a flight director. The flight director modes which provide tendency information to the pilot will take into account the identified downgraded handling capability of the aircraft under the current actuator failure situation.

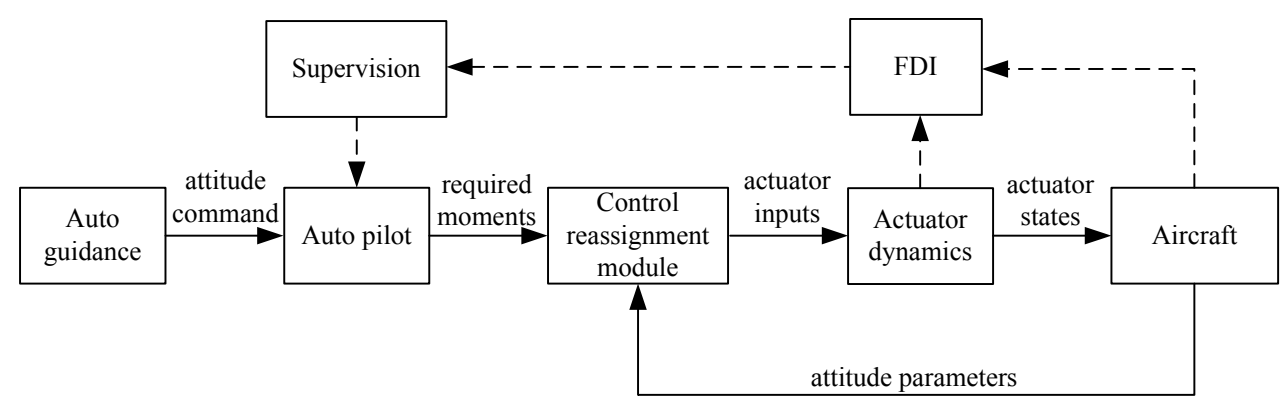

(a) fail-operational mode

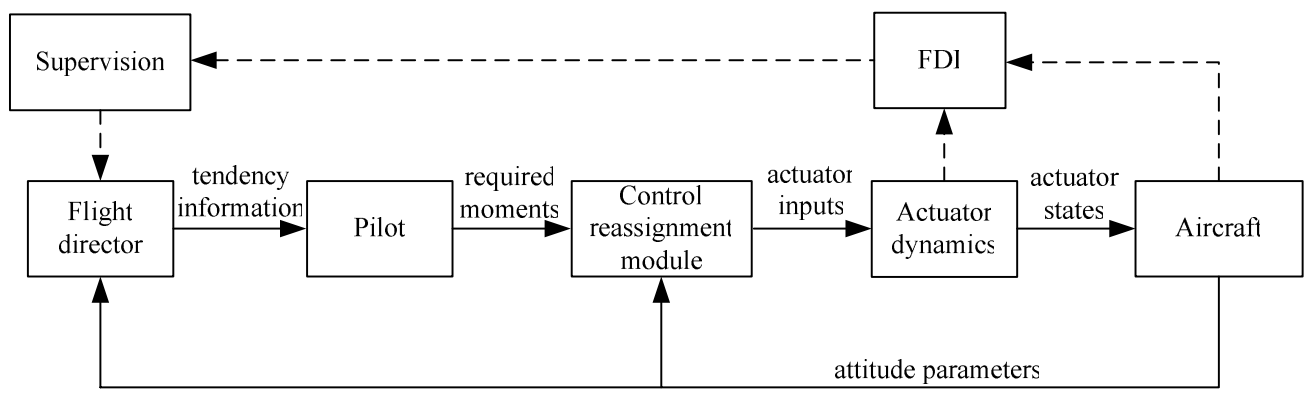

(b) fail-passive mode

Figure 1. Proposed modes for a fault tolerant control structure

This paper is organized as follows: In the next section, after considering the moment equations of a rigid aircraft, a global affine form describing the effectiveness of the aerodynamic actuators is introduced. In section III the constraints and limitations associated with the operation of these actuators are discussed. Then in section IV the MPC approach to assess the ability of the aircraft to perform in a standard way a given rotation maneuver is 
developed. Section V describes the dynamic neural network chosen to solve in an off-line environment the recurrent optimization problems associated with an MPC run. In section VI, preliminary results concerning three different fault scenarios treated with MPC approach are displayed and analyzed. Finally in section VII, a conclusion about the actual stage of the work is presented and the future steps to get an effective monitoring system contributing efficiently to a fault tolerant control architecture are discussed.

\section{Aircraft Fast Dynamics and Effectiveness of Aerodynamic Actuators}

\section{A. Aircraft rotation dynamics}

According to Newton's second law, the equation for the rotational movement of a rigid aircraft in the body-fixed axis system can be expressed as:

$$
\underline{M}=I_{m} \underline{\dot{\omega}}+\underline{\omega} \times\left(I_{m} \underline{\omega}\right)
$$

where $\underline{M}=\left(\begin{array}{lll}L & M & N\end{array}\right)^{\prime}, L, M, N$ are respectively the roll, pitch, and yaw aerodynamic torques, $I_{m}$ is the matrix of inertial moments, $\underline{\omega}$ is the inertial rotational velocity written in the body reference $(p, q, r)$ ' where $p$ is the roll rate, $q$ is the pitch rate and $r$ is the yaw rate, $\underline{\dot{\omega}}$ is the inertial rotational acceleration in the body-fixed axis system, $\times$ is the cross product operator.

The aerodynamic moments along each body axis are given by:

$$
L=\frac{1}{2} \rho V^{2} S l C_{l}, M=\frac{1}{2} \rho V^{2} S l C_{m}, N=\frac{1}{2} \rho V^{2} S l C_{n}
$$

where $C_{l}, C_{m}$ and $C_{n}$ are respectively the roll, pitch and yaw dimensionless aerodynamic coefficients. Here $V$ is the airspeed, $\rho$ is the density of air, $S$ and $l$ are respectively reference area and length specific to the considered aircraft.

The dimensionless coefficients of the main axis aerodynamic torques can in general be expressed such as:

$$
\begin{gathered}
C_{m}=C_{m 0}+C_{m \alpha} \alpha+C_{m q} q l / V+C_{m \delta t h s} \delta_{t h s}+\underline{C}_{m \delta q}{ }^{\prime} \underline{\delta}_{q} \\
C_{l}=C_{l 0}+C_{l \beta} \beta+C_{l p} p l / V+C_{l r} r l / V+\underline{C}_{l \delta p}, \underline{\delta}_{p}+\underline{C}_{l \delta r}{ }^{\prime} \underline{\delta}_{r} \\
C_{n}=C_{n 0}+C_{n \beta} \beta+C_{n p} p l / V+C_{n r} r l / V+\underline{C}_{n \delta p} \underline{\delta}_{p}+\underline{C}_{n \delta r} \underline{\delta}_{r}
\end{gathered}
$$

where $\alpha$ is the angle of attack, $\beta$ is the side slip angle, $\underline{\delta}_{p}, \underline{\delta}_{q}, \underline{\delta}_{r}$ are respectively the aileron, elevator and rudder deflections while $\delta_{t h s}$ is the deflection of the trimmable horizontal stabilizer, if any.

\section{B. Modeling the effectiveness of aerodynamic actuators}

Considering mainly the size of modern transportation aircraft as well as the reliability issue, these aircraft present in general a multiplicity of actuators to generate contributions to the roll, pitch and yaw moments. The effectiveness of these control surfaces appears through the contributions of their angular deflections to these moments through dimensionless coefficients as in relations (3.1), (3.2) and (3.3). According to the relationship between aerodynamic derivatives and aerodynamic torque, the expressions of the different aerodynamic torques generated by these control surfaces can be approximated by affine forms with respect to the corresponding deflections of the different aerodynamic actuators, so that we get expressions such as:

$$
\begin{gathered}
L(t)=L^{0}(t)+\sum_{i \in I^{L}} C_{i}^{L}(t) \delta_{i}(t) \\
M(t)=M^{0}(t)+\sum_{i \in I^{M}} C_{i}^{M}(t) \delta_{i}(t) \\
N(t)=N^{0}(t)+\sum_{i \in I^{N}} C_{i}^{N}(t) \delta_{i}(t)
\end{gathered}
$$


with $I=I^{L} \cup I^{M} \cup I^{N}$, where $I^{L}$ is the set of actuators generating some roll moment, $I^{N}$ is the set of actuators generating some yaw torque, while $I^{M}$ is the set of actuators generating pitch moments. Here the current values $L^{0}(t)$, $M^{0}(t)$ and $N^{0}(t)$ as well as $C_{i}^{L}(t), C_{i}^{M}(t)$ and $C_{i}^{N}(t)$ depend on the airspeed $V$, the flight level and on the values of $\alpha, \beta, p, q$ and $r$. Global aerodynamic torques generated by aircraft aerodynamic actuators can be rewritten in a global affine form as:

$$
\underline{M}=\underline{M}^{0}+C \cdot \underline{\delta} \quad \text { with } \underline{\delta} \in R^{|I|} \text { and } C \in R^{3 \times|I|}
$$

To illustrate the multiplicity of actuators on transportation aircraft, Fig. 2 displays, in the case of a A340 aircraft, the different aerodynamic surfaces of its wing which contribute mainly to the roll moment.

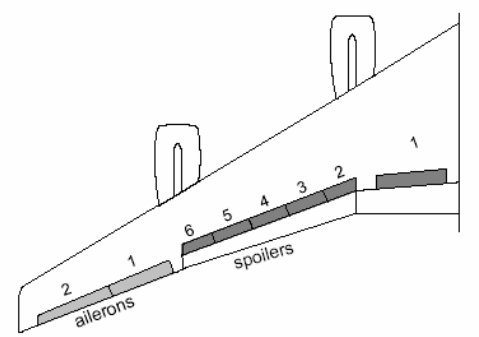

Figure 2. Example of Wing Actuators (A340)

\section{A reference model for rotational maneuver}

Substitute (5) into (1), we get the dynamics of rotational accelerations

$$
\underline{\dot{\omega}}=I_{m}^{-1}\left(\underline{M}^{0}+C \cdot \underline{\delta}-\underline{\omega} \times\left(I_{m} \underline{\omega}\right)\right)
$$

For the actuators whose deflection is present in the above relation, it is in general assumed that they present a linear domain in which they obey to first order dynamics such as:

$$
\underline{\dot{\delta}}=\Lambda(\underline{\delta}-\underline{\delta})
$$

where $\underline{\delta}_{c}$ is the control signal and $\Lambda$ is a constant diagonal matrix whose elements $\lambda_{i}$ are the reciprocals of time constants of each actuator $\left(\lambda_{i}=1 / \tau_{i}\right)$.

Considering relations (6) and (7), the rotation vector $\underline{\omega}$ is related to the control signal $\underline{\delta}_{c}$ by a rather complex nonlinear second order differential relation. Then it appears opportune to consider that the expected nominal rotational dynamics have to follow second order linear dynamics with adequate damping ratios, natural frequencies and constant time responses. So we consider such a reference model to be given by:

$$
\underline{\tilde{\tilde{\omega}}}+2 Z_{\omega} W_{\omega} \dot{\tilde{\tilde{\omega}}}+W_{\omega}^{2}\left(\underline{\tilde{\omega}}-\underline{\tilde{\omega}}_{c}\right)=0
$$

where $\underline{\tilde{\omega}}$ is nominal rotation rates vector and $\underline{\tilde{\omega}}_{c}$ is the desired rotational rates vector taken as input signal for this reference model. Here $Z_{\omega}, W_{\omega}$ are respectively the damping ratios and natural frequencies organized in $3 \times 3$ diagonal matrices. Let the resulting overall response time for these dynamics to step inputs along each axis be given by $T_{R}=\max \left\{T_{p}, T_{q}, T_{r}\right\}$. Here it is expected for feasibility reasons that the chosen time responses $T_{i}$ are many times the value of the time responses of the involved actuators in a fault free situation. When the remaining handling 
qualities do not allow an acceptable behaviour during the realization of the intended standard maneuver, the feasibility of downgraded reference manoeuvres can be investigated.

\section{Actuators Constraints and Limitations}

The deflection of each aerodynamic surface is subject to minimum and maximum bounds while its deflection rates are limited by the adopted actuator technology. Also, global physical constraints must be taken into account to ensure aircraft integrity especially when some actuators fail. These limitations are taken into consideration explicitly in the present study as they should be considered by any efficient fault tolerant control system.

\section{A. Actuators Position and Speed Limitations}

In general, the linear dynamics given in (7) are limited by position and rate constraints such as:

$$
\begin{array}{ll}
\delta_{i}^{\min } \leq \delta_{i} \leq \delta_{i}^{\max } & i \in I \\
\dot{\delta}_{i}^{\min } \leq \dot{\delta}_{i} \leq \dot{\delta}_{i}^{\max } & i \in I
\end{array}
$$

where $\delta_{i}^{\min }, \delta_{i}^{\max }, \dot{\delta}_{i}^{\min }$ and $\dot{\delta}_{i}^{\max }$ are the bounds and maximum deflection speed values. However, let $\tau_{i}$ be the time response of actuator $i$.

\section{B. Global Constraints}

Global constraints are in general related with structural considerations. It has been shown that total wing bending and torsion moments during maneuver can be written in an affine form: ${ }^{6}$

$$
\begin{aligned}
& M_{\text {bend }}(t)=A_{\text {bend }}(t)+\sum_{i \in I^{\text {wing }}} Y_{\text {bend }}^{i}(t) \delta_{i}(t) \\
& \text { and } \begin{aligned}
M_{\text {tors }}(t) & =A_{\text {tors }}(t)+\sum_{i \in I^{\text {wing }}} Y_{\text {tors }}^{i}(t) \delta_{i}(t)
\end{aligned}
\end{aligned}
$$

with $I^{\text {wing }} \subset I$ is the set of wing actuators contributing to the bending and the torsion moments, where $A_{b e n d}, Y_{b e n d}^{i}$, $A_{\text {tors }}$ and $Y_{\text {tors }}^{i}$ depend on the long run on the flight envelope parameters (airspeed and flight level) and on the short run on the current attitude parameters such as the angle of attack $\alpha$, the sideslip angle $\beta$, pitch and bank angles $\theta$ and $\phi$ and the roll, pitch and yaw rates $p, q$ and $r$.

To take into account the structural integrity of the aircraft, global constraints considering the wing bending and torsion moments can be introduced :

$$
\begin{aligned}
& A_{\text {bend }}(t)+\sum_{i \in I^{\text {wing }}} Y_{\text {bend }}^{i}(t) \delta_{i}(t) \leq M_{\text {bend }}^{\max } \\
& \text { and } \\
& A_{\text {tors }}(t)+\sum_{i \in I^{\text {wing }}} Y_{\text {tors }}^{i}(t) \delta_{i}(t) \leq M_{\text {tors }}^{\max }
\end{aligned}
$$

where $M_{\text {bend }}^{\max }$ and $M_{\text {tors }}^{\max }$ are maximum acceptable bending and torsion moments at the wing root. Here it is supposed that the satisfaction of these global constraints implies the satisfaction of local bending and torsion moment constraints. 


\section{Formulation of the Off-line Model Following Control Problem}

Model predictive control (MPC) is widely used today in control because of its ability to cope with hard constraints on controls and states as well as with modeling errors for the dynamics of the controlled process. ${ }^{7}$ In the flight control field, since actuator failures can result in new constraints, some authors have tried during the last decade to apply the MPC approach to design fault tolerant flight control systems. For example, in Ref. 8 a MPC based controller was proposed to tackle with the fault situation found in the accident of flight El Al 1862. In Refs. 9, 10 , actuator allocation has been considered for aircraft flight control using MPC without taking into account the actuator dynamics, while in Ref. 11 a MPC based dynamic control allocation algorithm considering actuator dynamics has been developed for a re-entry vehicle. However, MPC has proven most successful when controlling slow processes in the industrial field and more, the MPC approach implies at each time step the recurrent solution of an optimization problem whose size is roughly proportional to the size of the control horizon. Even if the resulting problem can be formulated as a standard linear quadratic optimization problem ${ }^{12,13}$ and with the current development of on-board computer capabilities, the direct adoption of this approach to perform flight dynamics online control, may appear rather questionable. If one of the resulting issues risen by MPC adoption is relative to possible numerical problems, another one is the quality of the resulting control performances which is not guaranteed, specially in the presence of fault, either successfully identified or not. However, to perform the present off-line study, these characteristics of the MPC approach are not limitative while its capability to take explicitly into account actuator constraints appears decisive.

\section{A. The adopted predictive models}

Equations (6) and (7) represent the complete rotation dynamics of the aircraft and their discretization according to Euler's method, provides the discrete expressions:

$$
\begin{aligned}
\underline{\omega}(k+1)= & \underline{\omega}(k)+I_{m}^{-1} T\left(\underline{M}^{0}+C \cdot \underline{\delta}(k)-\underline{\omega}(k) \times\left(I_{m} \underline{\omega}(k)\right)\right) \\
& \underline{\delta}(k+1)=\underline{\delta}(k)+\Lambda T\left(\underline{\delta}_{c}(k)-\underline{\delta}(k)\right)
\end{aligned}
$$

where $T$ is the sampling period.

Since when considering longitudinal or lateral maneuvers for a transportation aircraft, the cross product of relation (12) remains in general small, we adopted the following approximate linear model for the rotational dynamics:

$$
\underline{\omega}(k+1)=\underline{\omega}(k)+I_{m}^{-1} T\left(\underline{M}^{0}+C \cdot \underline{\delta}(k)\right)
$$

This simplification avoided us to have to cope within the MPC approach, with a recurrent non convex optimization problem, whose resolution may face numerical difficulties such as local non optimal minima. Then, combining relations (13) and (14), we get the following model which is a rough linear approximation of the discrete rotation dynamics:

$$
\underline{\omega}(k+1)=\underline{\omega}(k)+I_{m}^{-1} T\left(\underline{M}^{0}+C\left(I_{d}-T \Lambda\right) \underline{\delta}(k-1)+T \Lambda \underline{\delta}_{c}(k-1)\right)
$$

where $I_{d}$ is an identity matrix with size $|I| \times|I|$.

When considering the Model Predictive Approach the above discrete linear model will be used to predict the behaviour of the rotational dynamics over the receding control horizon. Then at current time $k$, the prediction of the rotation vectors $\underline{\omega}^{p}(k+i \mid k) i=1$ to $K$, where $K$ is the prediction horizon, given here by $K=\left\lceil T_{R} / T\right\rceil$, are such as:

$$
\underline{\omega}^{p}(k+1 \mid k)=\underline{\omega}(k)+I_{m}^{-1} T\left(\underline{M}^{0}+C\left(I_{d}-T \Lambda\right) \underline{\delta}(k-1)+T \Lambda \underline{\delta}^{*}(k-1)\right)
$$


and

$$
\underline{\omega}^{p}(k+i \mid k)=\underline{\omega}^{p}(k+i-1 \mid k)+I_{m}^{-1} T\left(\underline{M}^{0}+C \cdot \underline{\delta}^{p}(k+i-1 \mid k)\right) \quad i=2 \text { to } K
$$

with the predicted deflections of actuators at time $k$ given by:

$$
\underline{\delta}^{p}(k+i \mid k)=\underline{\delta}^{p}(k+i-1 \mid k)+\Lambda T\left(\underline{\delta}_{c}(k+i-1 \mid k)-\underline{\delta}^{p}(k+i-1 \mid k)\right) \quad i=1 \text { to } K-1
$$

Here at time $k, \underline{\delta}^{p}(k \mid k)=\underline{\delta}(k), \underline{\delta}^{p}(k+i \mid k) \quad i=1$ to $K-1$ is the predicted deflection value predicted at time $k$ for time $k+i, \underline{\delta}_{c}(k+i \mid k) \quad i=0$ to $K-2$ is the computed input solution for the recurrent optimization problem used by the MPC method, while $\underline{\delta}^{*}(k)=\underline{\delta}_{c}(k \mid k)$ is the adopted input solution for period $k$.

To proceed with the comparison of this predicted behaviour with the reference behaviour, the reference model of relation (8) must be also discretized. Then, according to Euler's method, the following second order difference equation is obtained:

$$
\underline{\tilde{\omega}}(k+1)-2\left(I_{d 1}-T Z_{\omega} W_{\omega}\right) \underline{\tilde{\omega}}(k)+\left(I_{d 1}-2 T Z_{\omega} W_{\omega}+T^{2} W_{\omega}^{2}\right) \underline{\tilde{\omega}}(k-1)-T^{2} W_{\omega}^{2} \underline{\tilde{\omega}}_{c}(k-1)=\underline{0}
$$

where $I_{d 1}$ is an identity matrix with size $3 \times 3$.

Since we considered only step responses starting at steady conditions, the predicted trajectory for the reference rotation vector is given at time $k$ by:

$$
\begin{aligned}
& \underline{\tilde{\omega}}(k+i)=2\left(I_{d 1}-T Z_{\omega} W_{\omega}\right) \underline{\tilde{\omega}}(k+i-1)-\left(I_{d 1}-2 T Z_{\omega} W_{\omega}+T^{2} W_{\omega}^{2}\right) \underline{\tilde{\omega}}(k+i-2)+T^{2} W_{\omega}^{2} \tilde{\omega}_{c} \quad i=2 \text { to } K \\
& \text { with } \quad \underline{\tilde{\omega}}(k)=\underline{\tilde{\omega}}(k-1)=\underline{0}, \underline{\tilde{\omega}}_{c}(k-1)=\underline{0}, \underline{\tilde{\omega}}_{c}(k+i)=\underline{\tilde{\omega}}_{c} \quad i=0 \text { to } K-1
\end{aligned}
$$

\section{B. Formulation of the recurrent optimization problem}

According to the considered problem and following the MPC technique, an objective function such as:

$$
J(k)=\sum_{i=1}^{K}\left\|\underline{\tilde{\omega}}(k+i)-\underline{\omega}^{p}(k+i \mid k)\right\|^{2}
$$

can be formulated at time $k$ where $\|\cdot\|^{2}$ is the $l_{2}$-norm. This function will be minimized with respect to the control inputs $\underline{\delta}_{c}(k+i), \mathrm{i}=0$ to $K-1$, while the predicted actuator deflections should satisfy positions and rates constraints. It is worth to observe that in the adopted formulation of the objective function, there is no need to introduce explicitly a penalty relative to control deviations since they are taken into account directly by the actuator constraints Also, no differentiated weightings are needed in the penalty relative to the deviation of the output from its nominal behaviour if no priority is introduced between elementary aircraft rotations when considering the manoeuvrability. Then, the problem of the choice of the quadratic weightings of the objective function which has to be faced with classical MPC is not an issue here.

The necessary discretization of constraints (9.1) and (9.2) leads to:

$$
\begin{gathered}
\underline{\delta}^{\min } \leq \underline{\delta}^{p}(k+i \mid k) \leq \underline{\delta}^{\max } \quad i=1 \text { to } K-1 \\
T \underline{\dot{\delta}}^{\min } \leq \underline{\delta}^{p}(k+i \mid k)-\underline{\delta}^{p}(k+i-1 \mid k) \leq T \underline{\dot{\delta}}^{\max } \quad i=2 \text { to } K-1
\end{gathered}
$$

Also the predicted actuator deflections should satisfy structural constraints such as: 


$$
A_{j}+Y_{j} \underline{\delta}^{p}(k+i \mid k) \leq M_{j}^{\max } \quad i=1 \text { to } K \quad j \in\{\text { bend,tors }\}
$$

while it is considered that the control input must also satisfy the extreme deflection constraints:

$$
\underline{\delta}^{\min } \leq \underline{\delta}_{c}(k+i \mid k) \leq \underline{\delta}^{\max } \quad i=0 \text { to } K-2
$$

Considering the predictive model of relation (16.3), when introducing the input parameters $\underline{\delta}_{c}$, these inequalities (19.1), (19.2), (20) can be transformed into affine inequalities with respect to these input parameters.

\section{The Proposed Neural Network Solver}

In this section, after displaying the standard formulation adopted for the recurrent linear optimization problem resulting from the MPC approach to solve the model following problem, the interest to adopt a dynamic neural network solver is discussed and its application to the present case is detailed.

\section{A. General formulation of the recurrent linear quadratic optimization problem}

The minimization of objective function $J(k)$ at time $k$ under constraints (19.1), (19.2), (20) and (21) leads to the resolution of a linear quadratic problem which can takes the form:

$$
\begin{gathered}
\min _{\underline{\underline{\delta}}_{c}} \underline{\underline{\delta}}_{c}(k)^{T} R(k) \underline{\underline{\delta}}(k)+\underline{\underline{s}}(k)^{T} \underline{\underline{\delta}}_{c}(k) \\
\text { with } A(k) \underline{\underline{\delta}}_{c}(k)+\underline{\underline{b}}(k) \leq \underline{0} \\
\text { where } \quad \underline{\underline{\delta}}^{\min } \leq \underline{\underline{\delta}}_{c}(k) \leq \underline{\underline{\delta}}^{\max } \\
{\left[\begin{array}{c}
\underline{\delta}_{c}(k \mid k) \\
\underline{\delta}_{c}(k+1 \mid k) \\
\vdots \\
\underline{\delta}_{c}(k+K-2 \mid k)
\end{array}\right] \in R^{|I| \times(K-1)}}
\end{gathered}
$$

In appendix the expressions of the current parameters $R(k), \underline{\underline{s}}(k), A(k), \underline{\underline{b}}(k), \underline{\underline{\delta}}^{\text {min }}$ and $\underline{\underline{\delta}}^{\text {max }}$ of the linear quadratic optimization problem considered at stage $k$ are given. There it can be checked that matrix $R(k)$ is semi definite positive.

\section{B. Fast neural solution for the linear quadratic optimization problem}

Many mathematical programming techniques ${ }^{12,13}$ are today available to solve convex optimization problems which adopt the general linear quadratic formulation displayed in (22), (23) and (24). In our case, according to the semi positive definiteness of the objective function and the considered linear inequality constraints, this problem is a convex mathematical programming problem and it can be solved efficiently by many existing mathematical programming techniques. Recently, methods such as active set methods and interior point methods have been applied to the actuator allocation problem formulated as a linearly constrained quadratic optimization problem. ${ }^{14,15}$ While the active set methods present some difficulty related with the combinatorial aspect of the active constraint set selection, the performance of the interior point methods is largely dependent of the initialization of the solution. More recently, solvers based on dynamic neural networks whose convergence speed is not compromised by the size of the problem have been proposed to solve the considered problem in the context of fault tolerant control. ${ }^{3}$ Thanks to the unchanged convergent speed, dynamic neural networks is a promising way to solve the allocation problem when the size of the problem is large, as it should be the case when adopting the MPC approach.

8

American Institute of Aeronautics and Astronautics 
The basic idea for solving an optimization problem using a tailored dynamic neural network is to make sure that it will converge exponentially and this towards an equilibrium point corresponding effectively to the optimal solution of the considered problem. Zhang in Ref. 16 proved the effectiveness of this approach. The exponential convergence is achieved by the choice of stable piecewise linear dynamics for the neural network and the convergence to the optimal solution is obtained by enforcing the equilibrium point to satisfy the primal-dual linear variational inequalities associated with the particular optimality conditions derived from the general Karush-KhunTucker optimality conditions. Zhang shown also that in the case in which the feasible set is empty the dynamic neural network is divergent.

The state of the corresponding dynamic neural network is defined by:

$$
\underline{x}=\left[\begin{array}{ll}
\underline{\delta}^{T} & \underline{u}^{T}
\end{array}\right]^{T}
$$

where $\underline{\underline{u}}$ is the dual variable vector associated to the inequality constraints (23) and its dynamics obeys to the piecewise differential equation:

$$
\frac{d \underline{x}}{d t}=\eta\left(I_{d 3}+N^{T}\right)\left\{P_{\Omega}(\underline{x}-(N \underline{x}+\underline{\rho}))-\underline{x}\right\}
$$

where $\eta$ is a positive parameter, $I_{d 3}$ is an identity matrix, $N$ and $\varrho$ are defined by:

$$
N=\left[\begin{array}{cc}
R & A^{T} \\
-A & 0
\end{array}\right] \quad \underline{\rho}=\left[\begin{array}{ll}
\underline{s}^{T} & -\underline{b}^{T}
\end{array}\right]^{T}
$$

Here $\Omega$ is the primal-dual feasible values set defined by:

$$
\Omega=\left\{\underline{x} \mid \underline{\mu}^{-} \leq \underline{x} \leq \underline{\mu}^{+}\right\} \quad \text { with } \quad \underline{\mu}^{-}=\left[\underline{\delta}^{\min } \underline{\underline{0}}\right]^{T} \quad \text { and } \quad \underline{\mu}^{+}=\left[\underline{\delta}^{\max } \triangleq\right]^{T}
$$

where $\Delta$ is a very large positive number and $P_{\Omega}$ is a projection operator over $\Omega$ such as:

$$
P_{\Omega}(\underline{x})=\underline{\mu}^{-} \quad \text { if } \underline{x} \leq \underline{\mu}^{-}, \quad P_{\Omega}(\underline{x})=\underline{x} \quad \text { if } \underline{\mu^{-}}<\underline{x}<\underline{\mu}^{+} \text {and } P_{\Omega}(\underline{x})=\underline{\mu}^{+} \quad \text { if } \underline{x} \geq \underline{\mu}^{+}
$$

Since the MPC approach is incremental over a receding time window, the initial state of the dynamic neural network at step $k+1$ has been taken equal to the solution of the linear quadratic problem of step $k$.

\section{Preliminary Results for the Proposed Approach}

Here a large transport aircraft with a reference flight speed of $120 \mathrm{~m} / \mathrm{s}$ has been considered. This aircraft has redundant actuators: two ailerons per wing, two elevators per side, and two rudders. These actuators are supposed to follow first-order dynamics with position and rate limitations. The values of these limitations and of the time constants of the actuator dynamics have been adopted from Ref. 17 and are displayed in Table 1. A flight dynamics simulator of this aircraft has been developed within the Simulink environment and used as the test bed.

Table I. Parameters of actuators under nominal condition

\begin{tabular}{|c|c|c|c|c|}
\hline Actuator & Number & Position limits & Rate limits & Time constants \\
\hline aileron & 4 & $-25^{\circ} \sim 25^{\circ}$ & $-25^{\circ} / \mathrm{s} \sim 25^{\circ} / \mathrm{s}$ & $0.15 \mathrm{~s}$ \\
\hline elevator & 4 & $-25^{\circ} \sim 10^{\circ}$ & $-15^{\circ} / \mathrm{s} \sim 15^{\circ} / \mathrm{s}$ & $0.15 \mathrm{~s}$ \\
\hline rudder & 2 & $-30^{\circ} \sim 30^{\circ}$ & $-25^{\circ} / \mathrm{s} \sim 25^{\circ} / \mathrm{s}$ & $0.3 \mathrm{~s}$ \\
\hline
\end{tabular}


The basic manoeuvre which has been considered here is a pure roll manoeuvre under different actuator failure situations. Figure 3 displays the roll input $\left(p_{\text {std }}\right)$ composed of two inverse steps and the output ( $\left.p_{\text {ref }}\right)$ resulting from the second order linear model (damping ratio $z_{p}=0.8$ and natural frequency $w_{n p}=2.5 \mathrm{rad} / \mathrm{s}$ ) chosen as reference.

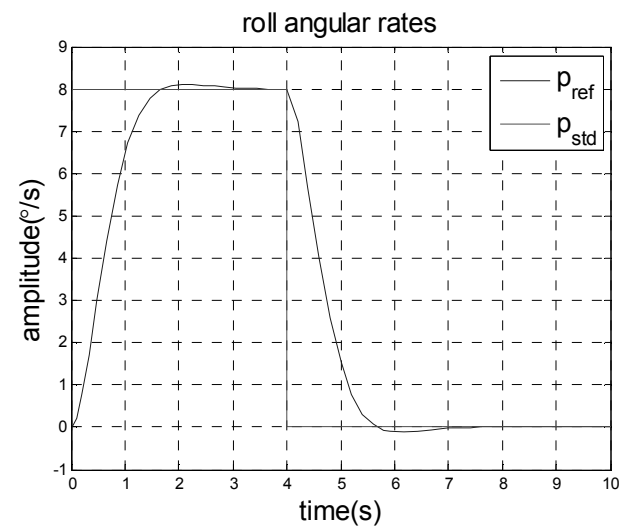

Figure 3. Standard roll input and reference output

The sampling period for discretization has been chosen equal to $0.05 \mathrm{~s}$ and since the response time of the reference manoeuvre is 2 seconds, the width of the sliding time window used in the MPC approach has been taken equal to $K=40$. The recurrent linear quadratic problem associated to MPC is then composed of 160 variables with 320 constraints. The dynamic neural network solver when tuned properly (choice of the value of $\eta$ ) produced each solution after a computation time close to $0.01 \mathrm{~s}$. This shows, that if the computation feasibility was the only criteria, MPC based flight control systems could be of interest.

The failure cases which are displayed here are relative to three situations:

Case a: the maximum deflection of the outer ailerons becomes limited to $10^{\circ}$.

Case b: the time constants of the outer ailerons become equal to $0.6 \mathrm{~s}$.

Case c: the outer ailerons are stuck at neutral position and the maximum deflection of the inner ailerons is limited to $15^{\circ}$.

Figures 4, 5 and 6 display the output behaviour obtained through the MPC process in these three cases.

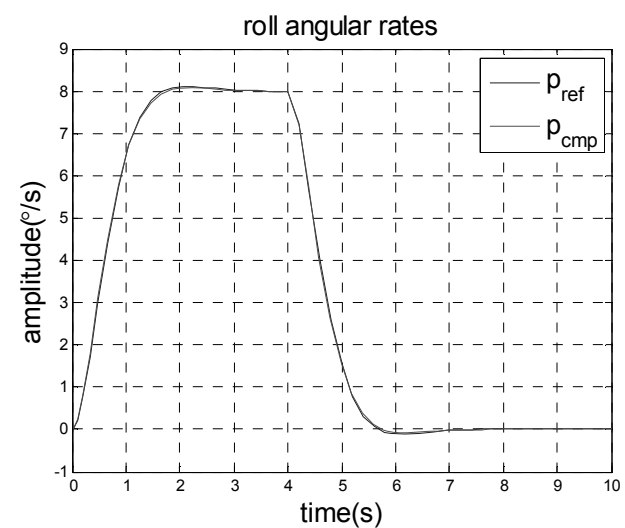

Figure 4. Case a: computed output and reference output 


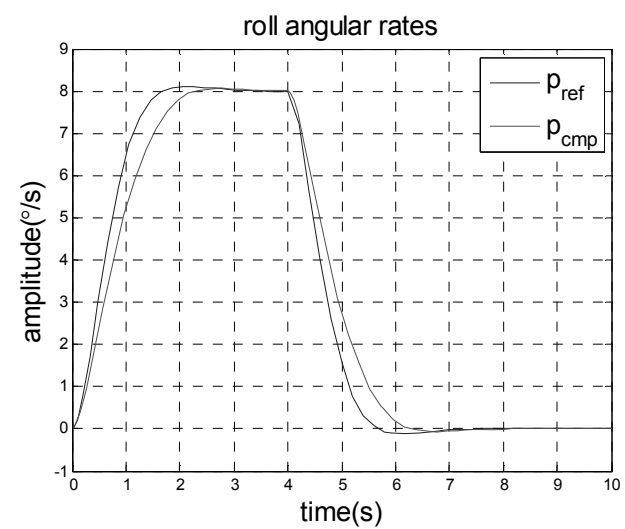

Figure 5. Case b: computed output and reference output

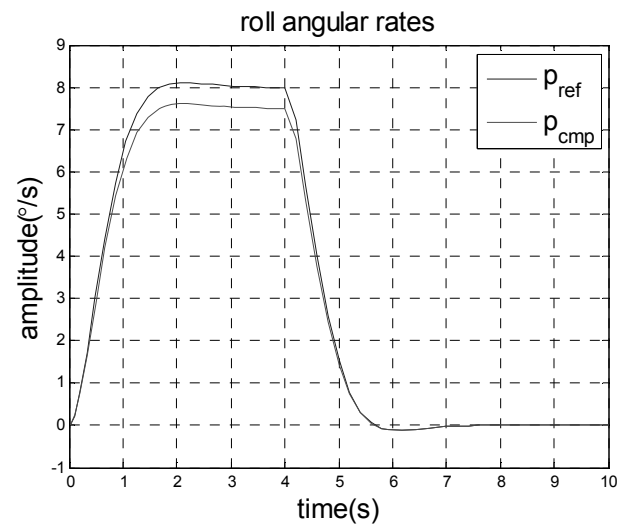

Figure 6. Case c: computed output and reference output

From the displayed results it appears that case a can be tackled as a fail-operational situation since the computed output is almost identical to the reference output while the other two cases should be tackled as fail-passive situations: in one case, case $b$, the response time of the basic roll loop is increased by $25 \%$ while in the other case, case $c$, the roll rate is limited to $7.5 \mathrm{deg} / \mathrm{s}$.

\section{Conclusion}

The objective of this study has been to contribute to the evaluation of the handling qualities of a transportation aircraft subject to aerodynamic actuator failures and this with the final aim to design more efficient fault tolerant flight control systems. The proposed approach to evaluate the remaining handling qualities of a transportation aircraft in an aerodynamic actuator failure situation has been based on the off-line resolution of a model following problem using a MPC approach coupled with a dynamic neural network solver.

The maneuverability issue in the present study has been limited to the control of the three rotations along the main axis of a transportation aircraft, since they are basic for stabilization, attitude control and further maneuvers. The preliminary numerical results corresponding to three simple failure scenarios show the computational feasibility of the proposed approach.

This approach can be extended to other maneuvers as well as to other types of actuators than those considered in this study, specially the engines, since the dynamics of the actuators have been taken into account. This provides a new tool to identify downgraded handling qualities resulting from actuator failures and then allows to build an exhaustive knowledge base which should be useful for the supervision of an efficient fault tolerant flight control system. 


\section{Appendix}

From relations (16.2) and (16.3), we get the expression of the predicted values for $\underline{\omega}^{p}(k+i \mid k), i=1$ to $K$ :

$$
\left[\begin{array}{c}
\underline{\omega}^{p}(k+1 \mid k) \\
\vdots \\
\underline{\omega}^{p}(k+K \mid k)
\end{array}\right]=\left[\begin{array}{c}
I_{d 1} \\
\vdots \\
I_{d 1}
\end{array}\right] \cdot \underline{\omega}^{p}(k+1 \mid k)+\left[\begin{array}{c}
0 \\
I_{d 1} \\
\vdots \\
(K-1) I_{d 1}
\end{array}\right] \cdot C_{0}+\left[\begin{array}{c}
0 \\
B_{3}\left(I_{d}-B_{4}\right) \\
\vdots \\
B_{3} \sum_{i=1}^{K-1}\left(I_{d}-B_{4}\right)^{i}
\end{array}\right] \cdot \underline{\delta}(k)+\left[\begin{array}{cccc}
0 & 0 & \cdots & 0 \\
B_{3} B_{4} & 0 & \cdots & 0 \\
\vdots & \vdots & \ddots & \vdots \\
B_{3}\left(\sum_{i=1}^{K-2}\left(I_{d}-B_{4}\right)^{i}\right) B_{4} & \vdots & \cdots & B_{3} B_{4}
\end{array}\right] \underline{\underline{\delta}}_{c}(k)(31)
$$

where $B_{3}=I_{m}^{-1} T C, B_{4}=\Lambda T$.

Relation (31) can be written in a more compact form as:

$$
\underline{\underline{\omega}}^{p}(k)=\underline{C}_{\delta}+H_{\delta} \underline{\underline{\delta}}_{c}(k)
$$

From relations (17.1), (17.2) and (17.3), we get the expression of the reference values for $\underline{\tilde{\omega}}(k+i), i=1$ to $K$ :

$$
\left[\begin{array}{c}
\underline{\tilde{\omega}}(k+1) \\
\vdots \\
\tilde{\tilde{\omega}}(k+K)
\end{array}\right]=\left[\begin{array}{c}
B_{7} B_{6} \\
\vdots \\
B_{7}\left(\sum_{i=0}^{K-1} B_{5}^{i}\right) B_{6}
\end{array}\right] \underline{\tilde{\omega}}_{c}
$$

where $B_{5}=\left[\begin{array}{cc}I_{d 1} & T I_{d 1} \\ -T W_{\omega}^{2} & \left(I_{d 1}-2 T Z_{\omega} W_{\omega}\right)\end{array}\right], B_{6}=\left[\begin{array}{c}0 \\ T W_{\omega}^{2}\end{array}\right], B_{7}=\left[\begin{array}{ll}I_{d 1} & 0\end{array}\right]$.

Relation (33) can be written in a more compact form as:

$$
\underline{\underline{\tilde{\omega}}}(k)=H_{\omega} \tilde{\omega}_{c}
$$

From expressions (18), (32) and (34), we get:

$$
R(k)=H_{\delta}^{T} H_{\delta} \quad \underline{\underline{s}}(k)=2 H_{\delta}^{T}\left(\underline{C}_{\delta}-H_{\omega} \tilde{\omega}_{c}\right)
$$

From relation (16.3), we get the expression of the predicted values for $\underline{\delta}^{p}(k+i \mid k), i=1$ to $K-1$ :

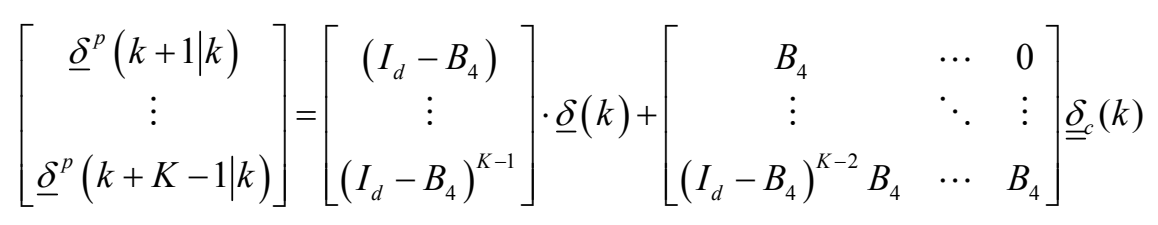

Relation (36) can be written in a more compact form as:

$$
\underline{\underline{\delta}}^{p}(k)=\underline{C}_{\delta 1} \underline{\delta}(k)+H_{\delta 1} \underline{\underline{\delta}}_{c}(k)
$$


From relations (19.1), (19.2), (20) and (37), we get:

$$
A(k)=\left[\begin{array}{c}
H_{\delta 1} \\
-H_{\delta 1} \\
D_{\delta} H_{\delta 1} \\
-D_{\delta} H_{\delta 1} \\
D_{Y_{j 1}} H_{\delta 1} \\
D_{Y_{j 2}} H_{\delta 1}
\end{array}\right], \underline{\underline{b}}(k)=\left[\begin{array}{c}
C_{\delta 1} \underline{\delta}(k)-\underline{\underline{\delta}}^{\max } \\
-C_{\delta 1} \underline{\delta}(k)+\underline{\underline{\delta}}^{\min } \\
D_{\delta} C_{\delta 1} \underline{\delta}(k)-T \dot{\underline{\delta}}^{\max } \\
-D_{\delta} C_{\delta 1} \underline{\delta}(k)+T \underline{\dot{\delta}}^{\min } \\
\underline{A}_{j 1}+D_{Y_{j 1}} C_{\delta 1} \underline{\delta}(k)-\underline{M}_{j 1}^{\max } \\
\underline{A}_{j 2}+D_{Y_{j 2}} C_{\delta 1} \underline{\delta}(k)-\underline{M}_{j 2}^{\max }
\end{array}\right]
$$

where $D_{\delta}=\left[\begin{array}{ccccc}-I_{d} & I_{d} & \cdots & 0 & 0 \\ \vdots & \vdots & \ddots & \vdots & \vdots \\ 0 & 0 & \cdots & -I_{d} & I_{d}\end{array}\right], D_{Y_{j 1}}=\left[\begin{array}{ccc}Y_{\text {bend }} & \cdots & 0 \\ \vdots & \ddots & \vdots \\ 0 & \cdots & Y_{\text {bend }}\end{array}\right], D_{Y_{j 2}}=\left[\begin{array}{ccc}Y_{\text {tors }} & \cdots & 0 \\ \vdots & \ddots & \vdots \\ 0 & \cdots & Y_{\text {tors }}\end{array}\right]$,

$$
\begin{gathered}
\underline{\underline{\delta}}^{\max }=\left[\begin{array}{c}
\underline{\delta}^{\max } \\
\vdots \\
\underline{\delta}^{\max }
\end{array}\right], \underline{\underline{\delta}}^{\min }=\left[\begin{array}{c}
\underline{\delta}^{\min } \\
\vdots \\
\underline{\delta}^{\min }
\end{array}\right], \underline{\dot{\delta}}^{\max }=\left[\begin{array}{c}
\underline{\delta}^{\max } \\
\vdots \\
\underline{\dot{\delta}}^{\max }
\end{array}\right], \underline{\underline{\delta}}^{\min }=\left[\begin{array}{c}
\underline{\delta}^{\min } \\
\vdots \\
\underline{\dot{\delta}}^{\min }
\end{array}\right], \\
\underline{A}_{j 1}=\left[\begin{array}{c}
A_{\text {bend }} \\
\vdots \\
A_{\text {bend }}
\end{array}\right], \underline{A}_{j 2}=\left[\begin{array}{c}
A_{\text {tors }} \\
\vdots \\
A_{\text {tors }}
\end{array}\right], \underline{M}_{j 1}^{\max }=\left[\begin{array}{c}
M_{\text {bend }}^{\max } \\
\vdots \\
M_{\text {bend }}^{\max }
\end{array}\right], \underline{M}_{j 2}^{\max }=\left[\begin{array}{c}
M_{\text {tors }}^{\max } \\
\vdots \\
M_{\text {tors }}^{\max }
\end{array}\right]
\end{gathered}
$$

\section{References}

${ }^{1}$ I. Hwang, S. Kim, Y. Kim, and C. E. Seah, "A Survey of Fault Detection, Isolation, and Reconfiguration Methods," IEEE Transactions on Control Systems Technology, vol. 18, pp. 636-653, 2010.

${ }^{2}$ Y. Han, S. Oh, B. Choi, D. Kwak, H. J. Kim, and Y. Kim, "Fault detection and identification of aircraft control surface using adaptive observer and input bias estimator," IET Control Theory \& Applications, vol. 6, pp. 1367-1387, 2012.

${ }^{3}$ L. Zhong and F. Mora-Camino, "Neural Networks Based Aircraft Fault Tolerant Control," presented at the 12th AIAA Aviation Technology, Integration, and Operations (ATIO) Conference and 14th AIAA/ISSMO Multidisciplinary Analysis and Optimization Conference, Indiana, USA, 2012.

${ }^{4}$ L. Zhong and F. Mora-Camino, "Aircraft Fault Tolerant Control Based on Active Set Method," presented at the 25th Chinese Control and Decision Conference, Guiyang, China, 2013.

${ }^{5}$ L. Zhong, H. Wu, H. Bouadi, and F. Mora-Camino, "Management of control channels under actuator failure: An optimization approach," presented at the 30th AIAA/IEEE Digital Avionics Systems Conference, Seattle, WA, USA, 2011.

${ }^{6}$ É. Roux, "Modèle de Masse Voilure: Avions de transport civil," Thesis PhD, pp. 349-349, 2006.

${ }^{7}$ D. Q. Mayne, J. B. Rawlings, C. V. Rao, and P. O. M. Scokaert, "Constrained model predictive control: Stability and optimality," Automatica, vol. 36, pp. 789-814, 2000.

${ }^{8}$ J. M. Maciejowski and C. N. Jones, "MPC fault-tolerant control case study: FLIGHT 1862," presented at the Proc. IFAC Safe Process Conf., 2003.

${ }^{9}$ S. Gaulocher, C. Cumer, and D. Alazard, "Aircraft load alleviation during maneuvers using optimal control surface combinations," Journal of Guidance, Control, and Dynamics, vol. 30, pp. 591-600, 2007.

${ }^{10}$ L. Lafourcade, C. Cumer, and C. Döll, "IMMUNE: Control Reallocation after Surface Failures Using Model Predictive Control," presented at the the 27th International Congress of the Aeronautical Sciences, Nice, France, 2010.

${ }^{11}$ Y. Luo, A. Serrani, S. Yurkovich, D. B. Doman, and M. W. Oppenheimer, "Model Predictive Dynamic Control Allocation with Actuator Dynamics," presented at the American Control Conference, 2004.

${ }^{12}$ D. G. Luenberger and Y. Ye, Linear and Nonlinear Programming: Springer, 2008. 
${ }^{13}$ S. Boyd and L. Vandenberghe, Convex Optimization. New York, USA: Cambridge University Press, 2004.

${ }^{14}$ O. Harkegard, "Efficient active set algorithms for solving constrained least squares problems in aircraft control allocation," presented at the the 41st IEEE Conference on Decision and Control, 2002.

${ }^{15}$ J. A. M. Petersen and M. Bodson, "Interior-point Algorithms for Control Allocation," Journal of guidance, control, and dynamics, vol. 28, pp. 471-480, 2005.

${ }^{16}$ Y. Zhang, "On the LVI-based Primal-Dual Neural Network for Solving Online Linear and Quadratic Programming Problems," presented at the American Control Conference, Porland, OR, USA, 2005.

${ }^{17}$ P. Lambrechts, S. Bennani, G. Looye, and D. Moormann, "The RCAM design challenge problem description," presented at the Robust Flight Control Lecture Notes in Control and Information Sciences, 1997. 